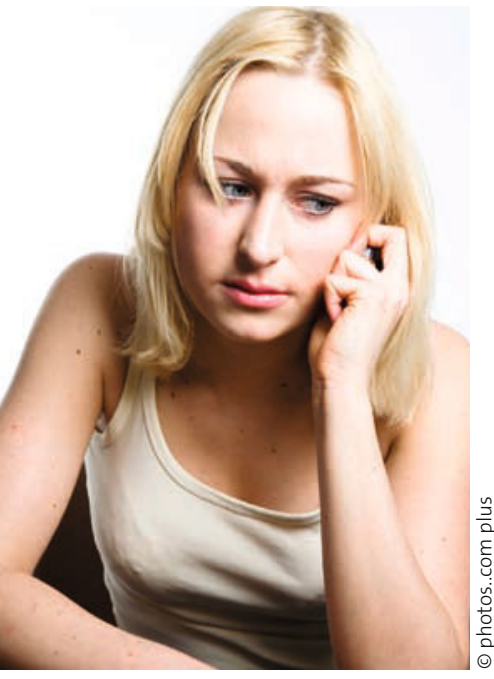

Ein Anruf zur falschen Zeit?
RUF NICHT AN, PAPA, ICH OVULIERE

\section{Wie der Eisprung das Verhalten von Frauen beeinflusst}

— Mit sich ankündigender Ovulation ändern Frauen unbewusst ihr Verhalten: Sie kleiden sich attraktiver und sind offener für die Avancen des anderen Geschlechts. Mit ihren Vätern indes kühlt der Kontakt in dieser Zeit merklich ab. Kommunikationsforscherin Martie Haselton von der Universität Kaliforniens (UCLA) und Kolleginnen untersuchten das Telefonverhalten von 48 Frauen im Alter von 18-22 Jahren. In den Tagen unmittelbar vor dem Eisprung riefen Frauen nur halb so oft ihren Vater an. Auch die Gesprächszeit halbierte sich auf 1,7 Minuten. Mit ihrer Mutter telefonierten sie an fruchtbaren Tagen viermal häufiger als mit dem Vater und mit 4,7 Minuten auch deutlich länger. Die Forscherinnen vermuten, dass Frauen in der Zeit ihrer größten Fruchtbarkeit unbewusst engen Kontakt mit engverwandten Männern meiden, um das Risiko der Inzucht zu minimieren.

Gleichzeitig erhöhen Frauen in dieser Zeit aber auch die Wahrscheinlichkeit, sich erfolgreich fortzupflanzen: Sie kleiden sich attraktiver und verändern ihre Stimmlage so, dass sie Männer auf bestimmte Weise anspricht. Zudem ziehen sie die Möglichkeit eines amourösen Abenteuers eher in Betracht als in Zeiten niedriger Fertilität. Ein besonderes Auge werfen sie auf Männer, deren Erscheinungsbild und Verhalten „echte Männlichkeit“ ausstrahlt.

Martie Haselton deutet die Studie so: „Unsere Alltagsentscheidungen sind immer noch eng an uralte Faktoren gebunden, die für Jahrtausende unsere Fortpflanzung und unser Überleben beeinflusst haben."

EH =

D. Lieberman et al. Psychological Science, 2010; DOI: 10.1177/0956797610390385
ANTIDEMENTIVE WIRKUNG

\section{Spaziergänge gegen das Vergessen}

- Patienten mit Alzheimer oder leichter kognitiver Beeinträchtigung $(\mathrm{MCl})$ können durch regelmäßige Spaziergänge den Gedächtnisverlust bremsen. Acht Kilometer pro Woche in gemächlichem Tempo genügen, um eine signifikante antidementive Wirkung zu erzielen. Das Gehen wirkt auch präventiv: Gesunde müssen knapp zehn Kilometer schaffen, um das Risiko einer sinkenden Gedächtnisleistung deutlich zu reduzieren. Das ist das Ergebnis einer Langzeitstudie mit 426 Freiwilligen im Alter von durchschnittlich 79 Jahren, darunter 127 Patienten mit MCI oder Alzheimer. Über eine Spanne von zehn Jahren wurden ihre Gehirnvolumina mittels MRT gemessen. Hippocampus, Vorder- und Schläfenlappen blieben bei denjenigen Patienten, die ausgedehntere Spaziergänge unternahmen, besser erhalten. Der Nutzen spiegelte sich auch im Mini-Mental-Status-Test (MMST) wider.

EO

Raji C, Jahrestagung der Radiologischen Gesellschaft Nordamerikas, 29.11.2010, Chicago

\section{AUCH BEI NORMALEM CHOLESTERINSPIEGEL \\ Xanthelasmen - Zeichen eines erhöhten Herzinfarktrisikos}

- Gelbliche Cholesterineinlagerungen rings um die Augen, sog. Xanthelasmen, sind nicht nur ein kosmetisches Problem. Sie weisen auch auf ein erhöhtes Risiko für schwere Herzerkrankungen hin. In einer dänischen Studie an 12939 Personen aus der Allgemeinbevölkerung war die Gefahr, im Lauf von 33 Jahren einen Herzinfarkt zu erleiden, um 51\% höher, wenn Xanthelasmen vorlagen ( $p<0,001$ ). Ein erhöhtes KHK-Risiko wurde bei 40\% der Probanden mit der Hautveränderung gefunden ( $p<0,0001)$. Das Gesamtsterberisiko war relativ um 17\% erhöht ( $p<0,0001)$.

Untersuchungen zum Zusammenhang von Xanthelasmen und kardiovaskulärem Risiko hat es in der Vergangenheit bereits gegeben. Neu ist, dass die Assoziation mit KHK und Herzinfarkt auch dann zu bestehen scheint, wenn erhöhte Serumlipide herausgerechnet wurden, sprich, es handelt sich um einen unabhängigen Marker. Die Ablagerungen entstehen, wenn Makro-

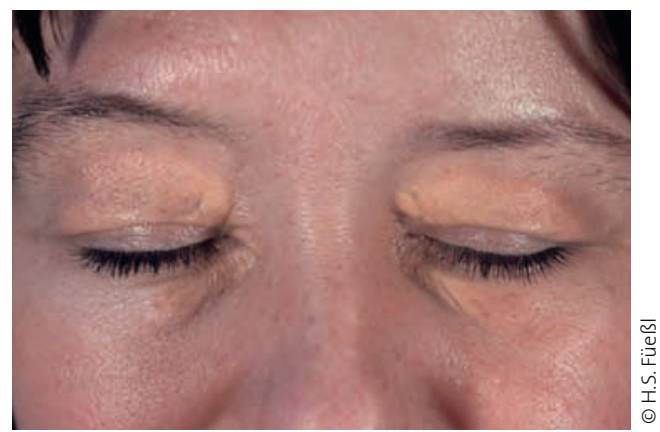

Ausgedehnte Xanthelasmen auf dem Oberlid bei Normocholesterinämie.

phagen Cholesterin aufgenommen haben und zu Schaumzellen werden. Allerdings hat fast jeder zweite Xanthelasmenträger normale Cholesterinwerte. Dies weist darauf hin, dass andere Faktoren (z.B. durchlässige Kapillaren, die Beschaffenheit der Makrophagen oder die Zusammensetzung der interzellulären Matrix) für die Entstehung von Xanthelasmen und gleichzeitig für eine KHK prädisponieren.

EO =

Christoffersen M, American Heart Association (AHA) 2010, Scientific Sessions, Chicago, November 2010 